\title{
NOTE ON COMPACT CLOSED CATEGORIES
}

\author{
B. J. DAY* \\ (Received 8 September 1976) \\ Communicated by R. H. Street
}

\begin{abstract}
Several categorical aspects of localisation to compact closed categories and free compact closed categories are discussed.
\end{abstract}

\section{Introduction}

The concept of a symmetric compact closed category was formalised by Kelly (1972). Generally speaking a compact bicategory is a bicategory in which each 1-cell has an adjoint. The details of this article can be followed through in this generality but we discuss, for simplicity, the "one-object" symmetric case over $\mathscr{E}$ ns.

By way of introduction we repeat the brief survey of Kelly (1972). A compact closed category is a symmetric monoidal category $(\mathscr{A}, \otimes, I)$ and a functor $*: \mathscr{A}^{\mathrm{oP}} \rightarrow \mathscr{A}$ and natural transformations $g_{A}: I \rightarrow A \otimes A *$, $h_{A}: A * \otimes A \rightarrow I$ such that $(1 \otimes h)(g \otimes 1)=1: A \rightarrow A \otimes A * \otimes A \rightarrow A$ and $(h \otimes 1)(1 \otimes g)=1: A * \rightarrow A * \otimes A \otimes A * \rightarrow A *$. Such a category is closed, with $[A, B]=A * \otimes B$; moreover, since adjoints are unique, we have $A \cong$ $A * *$ for all $A \in \mathscr{A}$. Conversely, a monoidal closed category is compact exactly when the canonical transformation $\kappa:[A, I] \otimes A \rightarrow[A, A]$ is an isomorphism whereupon $A *=[A, I], h$ is evaluation and $g$ is $I \rightarrow[A, A]=$ $[A ; A \otimes I]$ followed by $\kappa^{-1}$; as a consequence we have $A \cong[[A, I], I]$.

Perhaps the simplest non-trivial example of a compact closed category is the category of finite-dimensional vector spaces over a given field.

* The author gratefully acknowledges the support of a Postdoctoral Research Fellowship from the Australian Research Grants Committee. 


\section{Free compact closed cat egories and monadicity.}

Let $\mathscr{S M \mathscr { C }}$ denote the category of small symmetric monoidal closed categories and strict symmetric monoidal closed functors. Let $\mathscr{C} \mathscr{M C}$ denote the full subcategory of small compact closed categories.

\section{Proposition 1. The inclusion $\mathscr{C} \mathcal{M} \mathscr{C} \subset \mathscr{P M} \mathscr{C}$ has a left adjoint.}

Proof. We assign to each $\mathscr{A}=(\mathscr{A}, \otimes, I, \cdots) \in \mathscr{S} \mathscr{M} \mathscr{C}$ a universal compactification $C(\mathscr{A})$ together with a projection $P: \mathscr{A} \rightarrow C(\mathscr{A})$. Consider the class $K$ of transformations $\kappa:[A, I] \otimes B \rightarrow[A, B]$ and let $\bar{K}$ be its monoidal closure: $\bar{K}=\{A \otimes \kappa ; A \in \mathscr{A}$ and $\kappa \in K\}$. Then the effect of forming the symmetric monoidal category $\mathscr{A}\left(\bar{K}^{-1}\right)$ (Day (1973)) is equivalent to inverting the members of the class $S$ comprising the transformations $\sigma: B \otimes[A, C] \rightarrow[A, B \otimes C]$; this fact can be verified by simple coherent diagrams. It can also be seen that $S$ is in fact monoidal and that the transformations called Ten: $[A, B] \otimes[C, D] \rightarrow[A \otimes C, B \otimes D]$ are inverted. In particular the transformations $[A, I] \otimes[B, I] \rightarrow[A \otimes B, I]$ are inverted. Thus, if we write $A *$ for the image of $[A, I]$ under the projection $P: \mathscr{A} \rightarrow \mathscr{A}\left(S^{-1}\right)$, we have $A * \otimes B * \cong(A \otimes B) *$. This means that both the functors $\otimes: \mathscr{A} \times \mathscr{A} \rightarrow \mathscr{A}$ and $[-,-]: \mathscr{A}^{\mathrm{op}} \times \mathscr{A} \rightarrow \mathscr{A}$ factor to make $\mathscr{A}\left(S^{-1}\right)$ a compact closed category. It also induces on $P$ the structure of an $\mathscr{S} \mathscr{M C}$ morphism. We write $C(\mathscr{A})=\mathscr{A}\left(S^{-1}\right)$.

Remarks. The category $C(\mathscr{A})$ can be localised further to the "cancellative compactification" $C_{c}(\mathscr{A})$ of $\mathscr{A}$. This is formed by inverting, in addition to $S$, all the trasformations $A \otimes-:[B, C] \rightarrow[A \otimes B, A \otimes C]$. This process inverts all the transformations $A \otimes[A, B] \rightarrow B$ so that a cancellative compact closed category is a compact closed category for which $e:[A, B] \otimes A \rightarrow B$ is an isomorphism. In particular $A * \otimes A \cong A \otimes A * \cong$ $I$ in $C_{c}(\mathscr{A})$. The isomorphism classes of $C_{c}(\mathscr{A})$ form a preordered abelian group. When this preorder is replaced by the trivial preorder we obtain $K_{0}(\mathscr{A})$ where $K_{0}$ is left adjoint to $\mathscr{A} b \subset \mathscr{P} \mathscr{M} \mathscr{C}$. Thus $K_{0}(\mathscr{A})$ is universal for functions $f:|\mathscr{A}| \rightarrow G$. ( $G$ an abelian group) such that: (1) $A \cong B \Rightarrow f A=f B$, (2) $f(A \otimes B)=f A+f B,(3) f[A, B]=f B-f A$. If $\mathscr{A}$ is the free $\mathscr{P} \mathscr{M} \mathscr{C}$ category on a symmetric monoidal category $\mathcal{M}$ then $K_{0}(\mathscr{A})=K_{0}(\mathcal{M}, \bigotimes)$ (see Swan (1968); also see Conway (1976)).

Propostion 2. $\mathscr{C} \cdot \mathcal{M C}$ is monadic over $\mathscr{C}$ at and has all small limits and colimits.

Proof. The forgetful functor $U: \mathscr{C} M \mathscr{C} \rightarrow \mathscr{C}$ at has a left adjoint by monadicity of $\mathscr{S} \mathcal{M C}$ over $\mathscr{C}$ at (see Lambek (1969)) and Proposition 1. The 
same technique as used by Lambek (1969) can be used here to show that $U$ creates coequalisers of $U$-split pairs and coequalisers of reflective pairs; namely treat each $\sigma \in S$ and its inverse as a functor $\mathscr{A} \times \mathscr{A} \times \mathscr{A}^{\text {op }} \rightarrow \mathscr{E} n s^{2}$. Thus $\mathscr{C} \mathscr{M} \mathscr{C}$ is monadic over $\mathscr{C}$ at (by Beck's theorem; see Mac Lane (1971)) and has small colimits (by Linton (1969)).

Remark. Whilst $\mathscr{S} M \mathscr{C}$ is "clubable" over $\mathscr{C} a t, \mathscr{C} M \mathscr{C}$ is not (see Kelly (1972)).

\section{References}

J. H. Conway (1976), On numbers and games (Acad. Press, New York-London).

B. J. Day (1973), 'Note on monoidal localisation', Bull. Austral. Math. Soc., 8, 1-16.

S. Eilenberg and G. M. Kelly (1966), 'Closed categories'. Proc. Conference on Categorical Algebra, La Jolla 1965 (Springer-Verlag) 421-562.

G. M. Kelly (1972), Many-variable functorial calculus I, Coherence in Categories (Lecture Notes in Mathematics, 281, Springer-Verlag), 66-105.

J. Lambek (1969), Deductive systems and categories II, Category Theory, Homology Theory and their Applications I (Lecture Notes in Mathematics, 86, Springer-Verlag) 76-122.

F. E. J. Linton (1969), Coequalisers in categories of algebras, Seminar on Triples and Categorical Homology Theory (Lecture Notes in Mathematics, 80, Springer-Verlag) 75-90.

S. Mac Lane (1971), Categories for the working mathematician, GTM5 (Springer-Verlag, New York-Heidelberg-Berlin).

R. G. Swan (1968), Algebraic $K$-theory (Lecture Notes in Mathematics, 76, Springer-Verlag).

Department of Pure Mathematics,

University of Sydney,

N. S. W. 2006,

Australia. 